\title{
Multivariate symbol transfer entropy analysis on epileptic EEG
}

\author{
Qian-Qian Liu ${ }^{1}$, Jun Wang ${ }^{1,}{ }^{*}$ and Feng-Zhen $\mathrm{Hou}^{2, \mathrm{~b}}$ \\ ${ }^{1}$ Image Processing and Image Communications Key Lab, Nanjing Univ. of Posts \& Telecomm, \\ Nanjing 210003, China \\ ${ }^{2}$ School of Science, China Pharmaceutical University, Nanjing 210009, China \\ a E-mail: wangj@njupt.edu.cn, ${ }^{b}$ E-mail: houfz@cpu.edu.cn
}

Keywords: Complexity theory, multivariate symbolic transfer entropy, EEG signal

Abstract. Epilepsy is caused by abnormal synchronous discharge of neurons in the brain, which is the main basis for the diagnosis of epilepsy. Use of complexity theory to study the epileptic signal has become a hot spot. The symbolic transfer entropy can be used as a characteristic of epilepsy playing an increasingly important role in the study of epilepsy in EEG feature extraction. But symbolic transfer entropy is generally used to measure the dynamic characteristics and directional information between two variables and ignores the interaction between multivariate. In this paper, epileptic EEG signals is analyzed based on multivariate symbol transfer entropy. By choosing the lead signal and the signal length and analyzing the robustness, the method can be used to distinguish between normal and patients with epilepsy. It is proved the algorithm is robust and reliable. The findings will help clinical diagnosis.

\section{Introduction}

The main pathology of epilepsy is brain neuronal cell mass discharge abnormally and synchronously, diagnosis of epilepsy is currently in accordance with such discharge. It is significant that how to distinguish whether the patient's brain discharges or not, since epileptic patients showed no abnormality before it takes form and physical examination after the onset.

It has become a research hotspot to study epilepsy signal using the method of complexity. Entropy can be used as a characteristic of epilepsy playing an increasingly important role in the study of epilepsy in the extraction of EEG feature. There are many ways to measure the complexity of the signal currently, such as conditional entropy [1], and sample entropy [2]. Pseudo-relationship may be concluded only study the relationship between two variables, so it is great significant to study the characteristics of multivariate signal.

This paper is to analyse multivariate symbol transfer entropy EEG in epilepsy, which is use of the application of mutual condition information theory to look forward to distinguish between normal and epilepsy. The numerical results of the analysis found that the method can distinguish the normal and patients with epilepsy. Analysed the algorithm of noise immunity and found that the algorithm did not change much after superimposed white Gaussian noise and fully consistent trend, which shows that the algorithm is effective and reliable high robustness.

\section{Fundamental}

Multivariate transfer entropy. Transfer entropy [3] involves infinite vectors so it is hard to estimate reliably in high dimensions. To overcome this problem, the proposed transfer entropy decomposition.

Transfer entropy $I_{X \rightarrow Y}^{T E}=I\left(X_{t}^{-} ; Y_{t} \mid \mathbf{X}_{t}^{-} \backslash X_{t}^{-}\right)$is the reduction in uncertainty about $Y_{t}$ when learning the past of $X_{t}^{-}$. There are two infinite-dimensional parts in TE: $X_{t}^{-}$and $\mathbf{X}_{t}^{-} \backslash X_{t}^{-}$. We address the first by decomposing TE into contributions of individual lags of $X$ via the chain rule.

$$
I\left(X_{t}^{-} ; Y_{t} \mid \mathbf{X}_{t}^{-} \backslash X_{t}^{-}\right)=\sum_{\tau=1}^{\infty} I\left(X_{t-\tau} ; Y_{t} \mid \mathbf{X}_{t}^{-} \backslash X_{t}^{-}, X_{t-\tau}^{-}\right)
$$


Now the decisive step to escape the still infinite dimension of the condition in each term is done by utilizing the theory of graphical models [4].It is shown in Fig.1.

$$
I\left(X_{t-\tau} ; Y_{t} \mid \mathbf{X}_{t}^{-} \backslash X_{t}^{-}, X_{t-\tau}^{-}\right)=I\left(X_{t-\tau} ; Y_{t} \mid S_{Y_{t}, X_{t-\tau}}\right)
$$

The main idea stems from a Markov property that relates the separation of nodes in the graph to the conditional independences in the process. It implies that:

$$
I_{X \rightarrow Y}^{T E} \approx I_{X \rightarrow Y}^{D T E}=\sum_{\tau=1}^{\tau^{*}} I\left(X_{t-\tau} ; Y_{t} \mid S_{Y_{t}, X_{t \tau}}\right)
$$

Once the time series graph (shown in Fig.1) of the process is known, suitable sets $S_{Y_{r}, X_{t,}}$ can be determined from it and the TE can be estimated using only low-dimensional densities. The remaining infinite sum can be truncated at some finite $\tau^{*}$ since the terms typically decay exponentially with $\tau$.

past|present

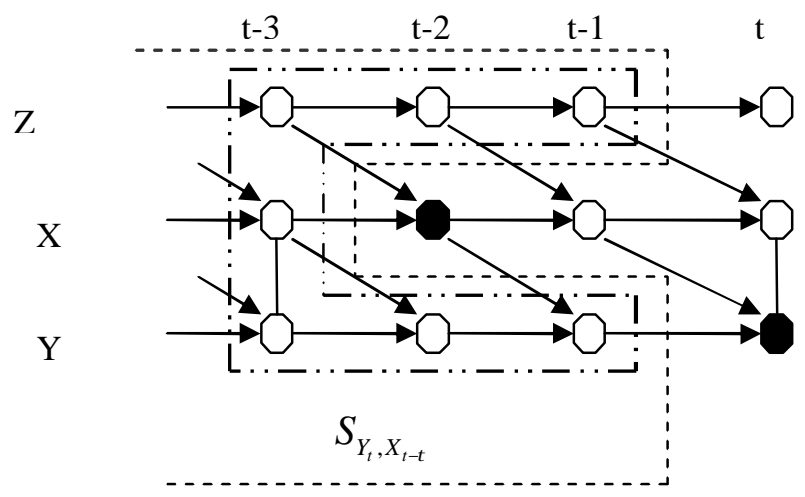

Fig. 1 Time series graph

Symbol of the original sequence. The Symbolic method [5] of original sequence is defined as follows:

$$
s_{i}\left(x_{i}\right)= \begin{cases}0: & u_{1}<x_{i} \leq(1+a) u_{1} \text { or }(1+a) u_{2} \leq x_{i}<u_{2} \\ 1: & (1+a) u_{1}<x_{i}<\infty \text { or }-\infty<x_{i}<(1+a) u_{2} \\ 2: & (1-a) u_{1}<x_{i} \leq u_{1} \text { or } u_{2} \leq x_{i}<(1-a) u_{2} \\ 3: & (1-a) u_{2} \leq x_{i} \leq(1-a) u_{1}\end{cases}
$$

$\mu_{1}$ and $\mu_{2}$ is the average of the original sequence is greater and less than zero sampled signal. In order to make the processed signal dynamics without losing time series is usually taken $\mathrm{a}=0.05$.

Multivariate symbol transfer entropy. Transfer entropy parameter has higher coordination requirements, so there has been transfer of entropy symbol [6].

Defined by multivariable transfer entropy [7] $I_{x \rightarrow r}^{T \pi} \approx I_{x \rightarrow r}^{o r r}=\sum_{I}\left(X_{t-\tau} ; Y_{i} \mid S_{\gamma, x_{x}}\right)$ can be deduced defined multivariate symbol transfer entropy. Upcoming sequence $X, Y, Z$ is converted into a sequence of symbols $S=\left\{s_{1}, s_{2}, \ldots, s_{i}, \ldots, s_{N}\right\} \quad, \quad J=\left\{j_{1}, j_{2}, \ldots, j_{i}, \ldots, j_{N}\right\} \quad, \quad K=\left\{k_{1}, k_{2}, \ldots, k_{i}, \ldots k_{N}\right\} \quad$ and $\quad s_{i} \in A(A=0,1,2,3) \quad$, $j_{i} \in A(A=0,1,2,3) ., k_{i} \in A(A=0,1,2,3)$.

Multivariate symbol transfer entropy is defined as:

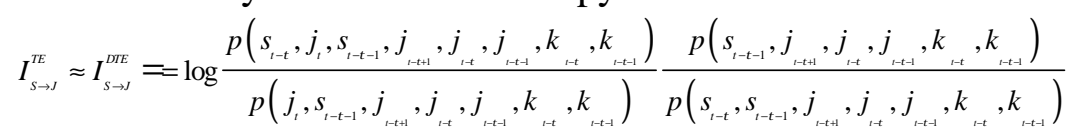




\section{Analysis of multivariate symbol transfer entropy}

Experimental data. This paper used the electrical signals that collected by the clinical diagnosis from general hospital of Nanjing military region. The database recorded multi-parameter EEG data (including 16 lead signal). The signal sampling period was $512 \mathrm{~Hz}$ and the recorded time was more than 1 minute. Take the lead signals F7、T5、T3, and calculate the transfer entropy of the symbol $F 7 \rightarrow T 5$ with $T 3$ known. We label the two groups of data as sample "normal" and sample "epilepsy".

Determine the selection of lead signal. For different lead group data, for each individual, with ten different point in time, select original sequence of the EEG signal that data length was $\mathrm{L}=80$ from the samples "normal" and "epilepsy". Take the average of transfer entropy of ten groups of each individual as final transfer entropy of the multivariate symbol of the individual. Then take average of transfer entropy of multiple symbols owned by normal and epilepsy. The result as fig 2, The horizontal axis represents the coordinates of meaning: 1-F7->T5|T3, 2-FP1->01|C3, 3-F3->P3|C3, 4-FP1->O2|C4, 5-F4->P4|C4, 6-F8->T6|T4, ste---> multivariate symbol transfer entropy. Lead symbol $F 7 \rightarrow T 5 \mid T 3$ had a good degree of differentiation.

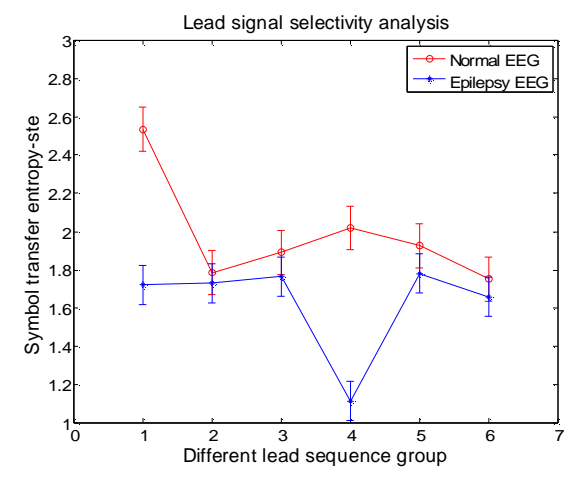

Fig. 2 Comparison of the symbolic transfer in total different lead groups

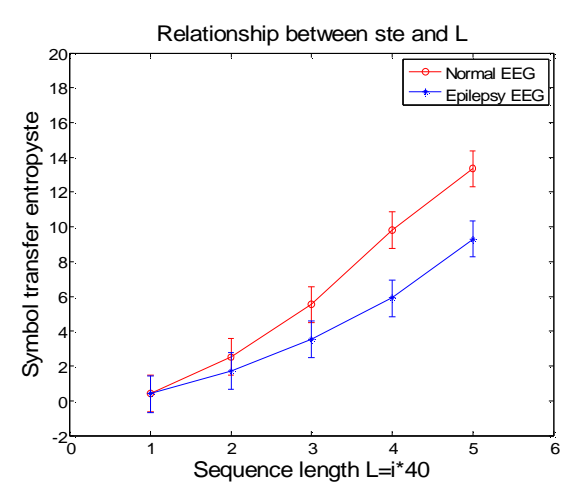

Fig.3 Relationship between transfer entropy data length $L$

The original EEG sequence were taken from each individual EEG sequence which come from sample "normal" and sample "epilepsy", take average of the multivariate symbol transfer entropy of normal and epilepsy patients. As shown in Fig.3, we take the data length $\mathrm{L}=160$.

Take a fixed length of $L=160$ original sequence of EEG from the sample "normal" and "epilepsy". Take average of multivariate TE of normal EEG and Epileptic respectively. The result is shown in Table 1.

Table 1 Multivariate symbolic transfer entropy of normal and epileptic

\begin{tabular}{|l|r|r|r|r|r|r|r|r|r|r|r|}
\hline Individual & 1 & 2 & 3 & 4 & 5 & 6 & 7 & 8 & 9 & 10 & mean \\
\hline Normal & 12.385 & 9.913 & 7.903 & 11.067 & 12.865 & 9.518 & 9.270 & 3.874 & 9.553 & 11.902 & 9.825 \\
\hline Epilepsy & 10.646 & 4.727 & 6.196 & 5.927 & 6.703 & 4.742 & 2.955 & 4.977 & 3.297 & 8.982 & 5.915 \\
\hline
\end{tabular}

Study of the multivariate transfer entropy in normal and epilepsy EEG, drawn in accordance with Table 1, the mean and variance of a diagram, shown in Fig.4

Use independent T-test analysis the results that obtained value of $\mathrm{T}=3.489, \mathrm{P}=0.002642<0.05$. This indicates the algorithm can distinguish the EEG of normal and patients with epilepsy significantly.

Robustness of the algorithm. To verify the robustness of the algorithm, taken the length of the data as $L=40 * i$ ( $i=1,2,3,4,5)$ of two types of brain signals the original sequence, superimposed white Gaussian noise from the sample "normal " and "epileptic ". The result is shown in Fig 5. 


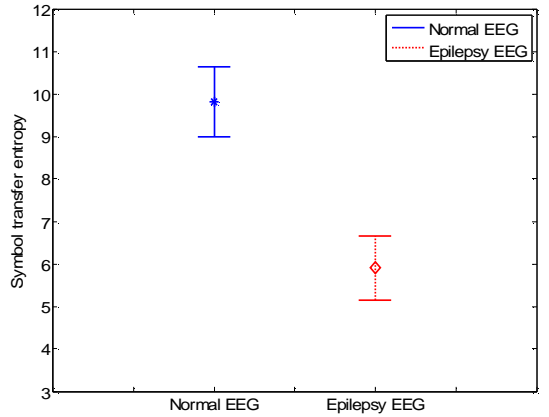

Fig. 4 Comparison of the symbolic tranfer entroy between normal and epilepsy

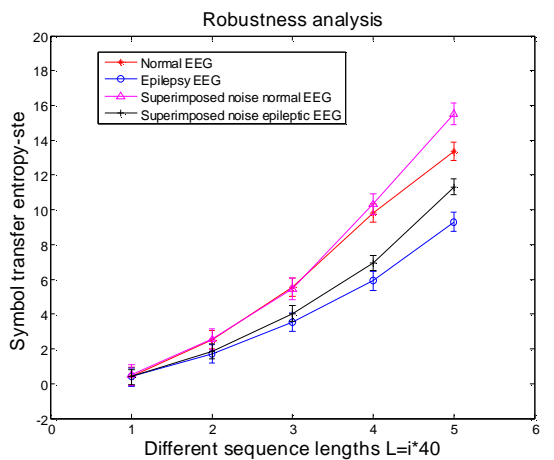

Fig.5 Robustness analysis of multi variable symblic transfer entropy algorithm

\section{Summary}

This article is based on multivariate analysis epileptic symbol transfer entropy, which is to study the relationship between several variables using conditional mutual information theory. Experimental results show that use of this method requires only a small sequence length can be analysed on the normal EEG and epilepsy, and can effectively distinguish normal EEG and epilepsy EEG. Use the method of symbolic to handle the original sequence of the EEG signal, it was found that superimposed noise on signal less affected by the noise. It illustrates the robustness of the algorithm. The findings will help clinical diagnosis.

\section{Acknowledgements}

Project supported by the National Natural Science Foundation of China (Grant Nos. 61271082, 61201029, 61401518), Jiangsu Provincial Key R \& D Program (Social Development) (Grant No. BE2015700), the Natural Science Foundation of Jiangsu Province (Grant No. BK20141432), the Foundation of Nanjing General Hospital of Nanjing Military Command (Grant No. 2014019) and the Fundamental Research Funds for the Central Universities (Grant No. FY2014LX0039).

\section{References}

[1] M. Javorka, B. Czippelova, L. Chladekova, Cardiovascular Control during Orthostatic and Mental Stress: Conditional Entropy Based Analysis, 2014 8th Conference of the European Study Group on Cardiovascular Oscillations (Esgco), (2014) 13-14.

[2] X. Jie, C. Rui, L. Li, Emotion recognition based on the sample entropy of EEG, Bio-Medical Materials and Engineering, 24 (2014) 1185-1192.

[3] S.Thomas, Measuring information transfer, Phys. Rev. Lett, 85 (2000) 461-464.

[4] E. Michael. Graphical modelling of multivariate time series. Probability Theory and Related Fields, 153 (2012) 233-268.

[5] E. J. Kostelich, The analysis of chaotic time-series data, Syst. Control Lett, 31(1997) 313-319.

[6] S. Matthaus, L. Klaus, Symbolic transfer entropy, Phys. Rev. Lett, 100(2008) 158101.

[7] R. Jakob, H. Jobst, P. Vladimir, and K. Jürgen, Escaping the Curse of Dimensionality in Estimating Multivariate Transfer Entropy, Phys. Rev. Lett, 108 (2012) 258701. 